\title{
Checking the optimality of entanglement witnesses: an application to structural physical approximations
}

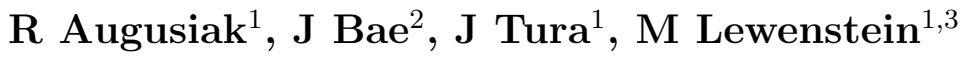 \\ ${ }^{1}$ ICFO-Institut de Ciencies Fotoniques, Mediterranean Technology Park, 08860 \\ Castelldefels (Barcelona), Spain \\ ${ }^{2}$ Center for Quantum Technologies, National University of Singapore, 3 Science \\ Drive 2, Singapore 117543 \\ ${ }^{3}$ ICREA-Institucio Catalana de Recerca i Estudis Avançats, Lluis Companys 23, \\ 08010 Barcelona, Spain
}

\begin{abstract}
In 2008, the conjecture that structural physical approximations to optimal entanglement witnesses are separable states (in general unnormalized) was posed. In an attempt to disprove it, in (Ha K-C and Kye S-H, Separable states with unique decompositions, arXiv:1210.1088v3), Ha and Kye proposed a decomposable entanglement witness whose SPA is entangled and argued that it is optimal. In this note, which is based on a comment to the latter work (Augusiak et al, Comment on "Separable states with unique decompositions", arXiv:1304.2040v1), we show, both analytically and numerically, that this entanglement witness is not optimal, and as such it is not a counterexample to the conjecture. To this end, we make use of a method for checking optimality of entanglement witnesses developed already in (Lewenstein et al 2000 Phys. Rev. A 62 052310), however, hardly exploited so far in the literature.
\end{abstract}




\section{Introduction and preliminaries}

Several years ago, in Ref. [1] some of us conjectured that a structural physical approximation (SPA) of an optimal positive map is entanglement breaking, or, equivalently, that SPA of an optimal entanglement witness is a separable state (in general unnormalized). Later, the conjecture was supported by numerous examples of witnesses (see, e.g., Refs. [2, 3, 4, 5] and also Ref. [6]), both decomposable and indecomposable, and even studied in the continuous variables case [5]. Quite surprisingly, in the indecomposable case it was recently disproved by Ha and Kye [7] (see also Ref. [8]), who found an example of an optimal indecomposable entanglement witness, whose structural physical approximation is entangled. Still, the conjecture remained unresolved in the decomposable case and later in an attempt to elucidate this, the same authors proposed in Ref. [9] a decomposable witness whose SPA is entangled and claimed it to be optimal. Had the latter been true, the conjecture would have been fully disproved.

The main aim of this note, which is a slightly modified version of the preprint [11] released as a comment to Ref. [9], is to show that the entanglement witness of Ref. [9] (see also Ref. [10]) is not optimal, and as such it is not a correct candidate to disprove the conjecture.

It should be stressed, however, that after our comment appeared on-line [11], the conjecture has eventually been disproved in the decomposable case by Chruściński and Sarbicki [12]. Nevertheless, we still find the optimality analysis presented in this note valuable for researchers working in entanglement theory. First, it is in general not an easy problem to analytically decide on optimality of an EW, if it does not have the so-called spanning property. Second, our note presents an instructive application of a powerful machinery for checking optimality of EWs developed already in Ref. [13], but rather hardly used in the literature.

Preliminaries. Before presenting our arguments, let us recall some background material. Let $\mathcal{H}=\mathbb{C}^{d}$ be a complex Hilbert space of dimension $d, M_{d}$ the set of $d \times d$ matrices of complex entries, and $\mathbb{1}_{d} \in M_{d}$ the identity matrix. A density operator acting on $\mathcal{H}$ is a positive semi-definite linear operator $\rho: \mathcal{H} \longrightarrow \mathcal{H}$ of unit trace.

Consider now a finite-dimensional product Hilbert space $\mathcal{H}_{A B}=\mathbb{C}^{d_{A}} \otimes \mathbb{C}^{d_{B}}$ and let $\mathcal{D}=\left\{\rho \in M_{d_{A}} \otimes M_{d_{B}} \mid \rho \geq 0, \operatorname{Tr} \rho=1\right\}$ denote the set of density operators acting on $\mathcal{H}_{A B}$. We say that $\rho \in \mathcal{D}$ is separable if, and only if it admits the following convex decomposition [14]:

$$
\rho=\sum_{i} p_{i} \rho_{A}^{i} \otimes \rho_{B}^{i}, \quad p_{i} \geq 0, \quad \sum_{i} p_{i}=1,
$$

where $\rho_{X}^{i}$ are density matrices acting on $\mathbb{C}^{d_{X}}(X=A, B)$. Otherwise we say that $\rho$ is entangled.

Entanglement not only fundamentally distinguishes between quantum and classical theories, but it is also a key resource for quantum information theory (QIT) (see [15]). 
Thus, its detection in composite quantum systems is one of the central problems in QIT (see [16]). A crucial method for this purpose, formulated in Ref. [17], exploits the fact that the set of separable states, denoted $\mathcal{D}_{\text {sep }}$, is closed and convex. Thus, it can be fully characterized by half-spaces whose normal vectors are non-positive semi-definite elements of the dual cone of $\mathcal{D}_{\text {sep }}, \mathcal{P}=\left\{W \in M_{d_{A}} \otimes M_{d_{B}} \mid \operatorname{Tr}(W \rho) \geq 0, \rho \in \mathcal{D}_{\text {sep }}\right\}$. Such operators form a nonconvex set, denoted $\mathcal{W}$, and, following Ref. [18], are called entanglement witnesses. Within this framework, $\rho \in \mathcal{D}_{\text {sep }}$ if, and only if, $\operatorname{Tr}(W \rho) \geq 0$ for any $W \in \mathcal{W}[17]$.

Clearly, not all EWs are necessary to detect all entangled states, and the first attempt to find the minimal set of EWs, which do the job, was made in Ref. [13], where the notion of optimal EW was introduced. To recall it, let us denote

$$
\Delta_{W}=\{\rho \in \mathcal{D} \mid \operatorname{Tr}(\rho W)<0\},
$$

and

$$
\Pi_{W}=\left\{|e, f\rangle \in \mathbb{C}^{d_{A}} \otimes \mathbb{C}^{d_{B}} \mid\langle e, f|W| e, f\rangle=0\right\} .
$$

Given $W_{1}, W_{2} \in \mathcal{W}, W_{1}$ is called finer than $W_{2}$ if $\Delta_{W_{2}} \subseteq \Delta_{W_{1}}$. If there is no witness finer that $W \in \mathcal{W}$, we call it optimal [13]. Alternatively speaking, a given $W \in \mathcal{W}$ is optimal iff for any $\lambda>0$ and any operator $P \geq 0$ having support orthogonal to $\Pi_{W}$, $W-\lambda P \notin \mathcal{W}$, i.e., the inequality

$$
\langle e, f|W-\lambda P| e, f\rangle \geq 0
$$

does not hold for some $|e, f\rangle$ (cf. Lemma 3 and Theorem 1, Ref. [13]). This implies a sufficient condition for optimality: if $\operatorname{span} \Pi_{W}=\mathcal{H}_{A B}$, then $W$ is optimal [13.

Geometrically, $\operatorname{Ext}(\mathcal{W}) \subset \operatorname{Opt}(\mathcal{W}) \subset \partial \mathcal{W}(\operatorname{cf} .[20])$, where $\operatorname{Ext}(\mathcal{W})$ and $\operatorname{Opt}(\mathcal{W})$ denote the sets of extremal (those generating extremal rays in $\mathcal{P}$ ) and optimal EWs, respectively, while $\partial \mathcal{W}$ stands for the boundary of $\mathcal{W}$. There exist, however, $W \in$ $\partial \mathcal{W} \backslash \operatorname{Opt}(\mathcal{W})$ and also $W \in \operatorname{Opt}(\mathcal{W}) \backslash \operatorname{Ext}(\mathcal{W})$, thus, in general, $\operatorname{Opt}(\mathcal{W}) \varsubsetneqq \partial \mathcal{W}$ and $\operatorname{Ext}(\mathcal{W}) \varsubsetneqq \operatorname{Opt}(\mathcal{W})$. As an illustrative example of $W \in \partial \mathcal{W} \backslash \operatorname{Opt}(\mathcal{W})$ consider a line segment $W(p)=p W_{+}+(1-p) W_{-} \in M_{2} \otimes M_{2}$ with $0 \leq p \leq 1$, where $W_{ \pm}=\left|\psi_{ \pm}\right\rangle\left\langle\left.\psi_{ \pm}\right|^{T_{B}} \in \operatorname{Ext}(\mathcal{W})\right.$ with $\left.\mid \psi_{ \pm}\right\rangle=(1 / \sqrt{2})(|00\rangle \pm|11\rangle)$. For any $p \neq 1 / 2$, $W(p)$ is an $\mathrm{EW}$, while $W(1 / 2) \geq 0$, and therefore $W(p)$ are not optimal for any $p \neq 0,1$. To prove that $W(p) \in \partial \mathcal{W}$ for any $0 \leq p \leq 1$, it is enough to notice that $W(p)-\epsilon\left|\phi_{+}\right\rangle\left\langle\left.\phi_{+}\right|^{T_{B}} \notin \mathcal{W}\right.$ for any $\epsilon>0$ with $\left.\mid \phi_{+}\right\rangle=(1 / \sqrt{2})(|01\rangle+|10\rangle)$. Then, as an example of $W \in \operatorname{Opt}(\mathcal{W}) \backslash \operatorname{Ext}(\mathcal{W})$ consider a decomposable $\ddagger$ witness $W=Q^{T_{A}}$ with $Q \in M_{2} \otimes M_{d}(d \geq 2)$, such that $Q \geq 0$ and $\operatorname{supp}(Q)$ is a completely entangled subspace§. Any such EW is optimal [19], still it is not extremal provided $\operatorname{rank}(Q)>1$.

$\ddagger$ We call $W \in \mathcal{W}$ decomposable if $W=P+Q^{T_{B}}$ with $P, Q$ being two positive semi-definite operators and $T_{B}$ denoting the partial transposition with respect to the second Hilbert space $\mathbb{C}^{d_{B}}$ (the partial transposition with respect to the first subsystem can equivalently be taken). Otherwise $W$ is called indecomposable. Notice that originally the notion of decomposability was formulated for positive maps in [28, 29] and later translated via the Choi-Jamiołkowski isomorphism to EWs.

$\S$ A subspace of $\mathbb{C}^{d_{A}} \otimes \mathbb{C}^{d_{B}}$ is called completely entangled if it contains no product vectors (for more information about these objects see, e.g., Ref. [30]). 
Noticeably, although extremal or even exposed EWs form proper subsets of Opt $(\mathcal{W})$, they detect all entangled states (see Refs. [20, 21, 22]). Yet, the definition of optimal EWs is operational in the sense that it can be recast to an efficient algorithm bringing any witness to an optimal one [13]. As such, optimal EWs remain a crucial tool in entanglement theory.

The above concepts can be recast in terms of positive maps. Recall that the Choi-Jamiołkowski isomorphism [23, 24] (see also Ref. [25]) establishes the equivalence between the set $\mathcal{L}\left(M_{d}, M_{d^{\prime}}\right)$ of linear maps from $M_{d}$ to $M_{d^{\prime}}$ and $M_{d} \otimes M_{d^{\prime}}$. Within this pairing $\mathcal{P}$ and $\mathcal{W}$ correspond to the convex cone of positive maps and its subset of those maps that are not completely positive, respectively. Accordingly, a positive map is called optimal if its corresponding EW is optimal. Then, any element of $\mathcal{D}_{\text {sep }}$ is isomorphic to an entanglement breaking channel [26] (see also [27]), that is a completely positive map $\Lambda: M_{d} \longrightarrow M_{d^{\prime}}$ such that (i) $(I \otimes \Lambda)[\rho] \in \mathcal{D}_{\text {sep }}$ for all $\rho \in \mathcal{D}$ and (ii) $\operatorname{Tr}[\Lambda(X)]=\operatorname{Tr} X$ for any $X \in M_{d}$ (trace-preservicity).

In terms of positive maps the above separability criterion reads [17]: $\rho \in \mathcal{D}_{\text {sep }}$ iff $(I \otimes \Lambda)[\rho] \geq 0$ for any positive map $\Lambda: M_{d_{B}} \longrightarrow M_{d_{A}}$. A positive map gives a more powerful necessary condition for separability than the corresponding $\mathrm{EW}$ : the best known example is given by the transposition map, which detects all two-qubit and qubit-qutrit entangled states [17], whereas the associated EW only some of them. However, the main drawback of positive maps in comparison to EWs is that they cannot be realized in physical experiments, since positive but not $\mathrm{CP}$ maps do not represent physical processes. The structural physical approximation (SPA) 31] is a method that allows one to overcome this problem by mixing a positive map $\Lambda$ with the completely depolarizing channel defined as $D(X)=\operatorname{Tr}(X) \mathbb{1}_{d} / d$ for $X \in M_{d}$. Clearly, for any positive map $\Lambda$ there exists $p_{*} \in(0,1)$ such that for any $p \in\left[0, p_{*}\right]$ the linear map $\Lambda(p)=p \Lambda+(1-p) D$ is completely positive, and as such represents a physical process. For the largest $p=p_{*}$ for which this is the case, we call $\Lambda\left(p_{*}\right)$ the SPA of $\Lambda$. Notice that via the Choi-Jamiołkowski isomorphism, one can translate the notion of SPA to EWs: given $W \in \mathcal{W}$, the operator

$$
W\left(p_{*}\right)=p_{*} W+\left(1-p_{*}\right) \frac{\mathbb{1}_{d_{A} d_{B}}}{d_{A} d_{B}} .
$$

with $p_{*}=1 /\left(1+d_{A} d_{B}\left|\lambda_{\min }\right|\right)$, where $\lambda_{\min }<0$ is the minimal eigenvalue of $W$, is called the SPA of $W$.

\section{A method to test optimality of EWs}

Let us now sketch the method developed in Ref. [13], that we will later exploit to demonstrate that the EW of Refs. [9, 10] is not optimal. To this end, consider a decomposable $W \in \mathcal{W}$ and let $\epsilon$ be a positive number and $P$ a positive semi-definite operator acting on $\mathcal{H}_{A B}$ whose support is orthogonal to $\Pi_{W}$. We assume that such a nonzero $P$ exists; otherwise, if there is no such $P$, or, equivalently span $\Pi_{W}=\mathcal{H}_{A B}$, the 
witness is optimal (see Sec. 1). Clearly, for all normalized product vectors $|e, f\rangle \in \mathcal{H}_{A B}$ satisfying

$$
\langle e, f|W| e, f\rangle>\epsilon
$$

there always exists $\lambda>0$ (e.g., $\lambda<\epsilon / M$ with $M=\max _{|e, f\rangle}\langle e, f|P| e, f\rangle$ ) such that the inequality (4) is fulfilled. It is obvious that the same holds for any $\epsilon>0$, even arbitrarily small, and corresponding product vectors obeying (6). One then needs to check whether (4) remains satisfied by those product vectors which do not obey (6), i.e., product vectors for which $\langle e, f|W| e, f\rangle \leq \epsilon$, where $\epsilon$ can be considered a free parameter that can be set arbitrarily small. A product vector $|e, f\rangle$ obeying the latter inequality must then be "close" (in the norm induced by the scalar product in $\mathcal{H}$, i.e., $\||\psi\rangle \|=\sqrt{\langle\psi \mid \psi\rangle}$ ) to one of the elements of $\Pi_{W}$. To see it in a more explicit way, consider a sequence of product vectors $\left|e_{n}, f_{n}\right\rangle$ such that $\left\langle e_{n}, f_{n}|W| e_{n}, f_{n}\right\rangle \leq \epsilon_{n}$ with $\epsilon_{n} \rightarrow 0$ as $n \rightarrow \infty$. Assume that elements $\left|e_{n}, f_{n}\right\rangle$ in the sequence do not converge, in the norm $\|\cdot\|$ to any element in $\Pi_{W}$ as $n \rightarrow \infty$. However, since the set of normalized vectors in a finite-dimensional Hilbert space is compact, the sequence $\left|e_{n}, f_{n}\right\rangle$ contains a subsequence that converges to some nonzero product vector $\left|e^{\prime}, f^{\prime}\right\rangle$ satisfying $\left\langle e^{\prime}, f^{\prime}|W| e^{\prime}, f^{\prime}\right\rangle=0$. By assumption $\left|e^{\prime}, f^{\prime}\right\rangle \notin \Pi_{W}$, which contradicts the fact that $\Pi_{W}$ contains all product vectors satisfying the condition in Eq. (3).

Let us now, for simplicity, restrict to the case $\mathcal{H}=\mathbb{C}^{3} \otimes \mathbb{C}^{3}$, but the method that follows can be straightforwardly generalized to any $d_{A}$ and $d_{B}$. It follows from what we have just said that for any product vector $|e, f\rangle \in \mathbb{C}^{3} \otimes \mathbb{C}^{3}$ such that $\langle e, f|W| e, f\rangle \leq \epsilon$ there is a normalized $\left|e_{0}, f_{0}\right\rangle \in \Pi_{W}$ such that local components of $|e, f\rangle$ can be written as

$$
|e\rangle=\frac{1}{\sqrt{1+\left|\delta_{1}\right|^{2}+\left|\delta_{2}\right|^{2}}}\left(\left|e_{0}\right\rangle+\delta_{1}\left|e_{1}\right\rangle+\delta_{2}\left|e_{2}\right\rangle\right)
$$

and

$$
|f\rangle=\frac{1}{\sqrt{1+\left|\omega_{1}\right|^{2}+\left|\omega_{2}\right|^{2}}}\left(\left|f_{0}\right\rangle+\omega_{1}\left|f_{1}\right\rangle+\omega_{2}\left|f_{2}\right\rangle\right)
$$

where $\left|e_{i}\right\rangle$ and $\left|f_{i}\right\rangle(i=1,2)$ are normalized vectors orthogonal to $\left|e_{0}\right\rangle$ and $\left|f_{0}\right\rangle$, respectively, while $\delta_{i}$, and $\omega_{i}(i=1,2)$ are complex numbers of vanishing absolute values for $\epsilon \rightarrow 0$.

By inserting Eqs. (7) and (8) into Eq. (6), one arrives at

$$
\langle e, f|W| e, f\rangle=\sum_{i=0}^{4} A_{i}(W),
$$

where by $A_{i}(W)$ we have denoted terms of the $i$ th (total) degree in the variables $\delta_{j}$, and $\omega_{j}(j=1,2)$. From the fact that $\left|e_{0}, f_{0}\right\rangle \in \Pi_{W}$, and that the matrices $\left\langle e_{0}|W| e_{0}\right\rangle=\operatorname{Tr}_{A}\left[\left(\left|e_{0}\right\rangle\left\langle e_{0}\right| \otimes \mathbb{1}_{3}\right) W\right]$ and $\left\langle f_{0}|W| f_{0}\right\rangle=\operatorname{Tr}_{B}\left[\left(\mathbb{1}_{3} \otimes\left|f_{0}\right\rangle f_{0} \mid\right) W\right]$ are positive semi-definite, it follows that $A_{0}(W)=A_{1}(W)=0$ (analogously, $A_{0}(P)=A_{1}(P)=0$ for any $P \geq 0$ ), and the first non-vanishing term in Eq. (9) can be $A_{2}(W)$. On the other hand, $A_{3}(W)$ and $A_{4}(W)$ can be made arbitrarily small by appropriately adjusting $\epsilon$, 
Checking the optimality of entanglement witnesses: an application to ...

which also means that $A_{2}(W) \geq 0$. It is then clear that if for any element of $\Pi_{W}$, $A_{2}(W)$ is always positive except for the case $\delta_{1}=\delta_{2}=\omega_{1}=\omega_{2}=0$, then for $P \geq 0$ one can adjust $\lambda$ such that the condition (4) is fulfilled for any $|e, f\rangle$. In this way we have arrived at the sufficient criterion for an EW $W$ to be nonoptimal: if $\operatorname{span} \Pi_{W} \varsubsetneqq \mathcal{H}_{A B}$ and $A_{2}(W)>0$ for any element of $\Pi_{W}$ unless $\delta_{1}=\delta_{2}=\omega_{1}=\omega_{2}=0$, then $W$ is not optimal.

\section{A proof that the decomposable EW of Ref. [9] is not optimal}

We can now pass to the decomposable EW introduced by $\mathrm{Ha}$ and Kye [9, 10]. It acts on $\mathcal{H}_{A B}=\mathbb{C}^{3} \otimes \mathbb{C}^{3}$ and is given by

$$
\begin{aligned}
W_{\theta, b} & =\left|w_{0}\right\rangle\left\langle\left. w_{0}\right|^{T_{A}}+\frac{1}{b} \sum_{i=1}^{3} \mid w_{i}\right\rangle\left\langle\left. w_{i}\right|^{T_{A}}\right. \\
& =\left(\begin{array}{ccccccccc}
1 & 0 & 0 & 0 & \mathrm{e}^{\dot{\mathrm{i}} \theta} & 0 & 0 & 0 & \mathrm{e}^{-\dot{\mathrm{i}} \theta} \\
0 & b & 0 & 1 & 0 & 0 & 0 & 0 & 0 \\
0 & 0 & \frac{1}{b} & 0 & 0 & 0 & 1 & 0 & 0 \\
0 & 1 & 0 & \frac{1}{b} & 0 & 0 & 0 & 0 & 0 \\
\mathrm{e}^{-\dot{\mathrm{i}} \theta} & 0 & 0 & 0 & 1 & 0 & 0 & 0 & \mathrm{e}^{\mathrm{i} \theta} \\
0 & 0 & 0 & 0 & 0 & b & 0 & 1 & 0 \\
0 & 0 & 1 & 0 & 0 & 0 & b & 0 & 0 \\
0 & 0 & 0 & 0 & 0 & 1 & 0 & \frac{1}{b} & 0 \\
\mathrm{e}^{\dot{\mathrm{i}} \theta} & 0 & 0 & 0 & \mathrm{e}^{-\mathrm{i} \theta} & 0 & 0 & 0 & 1
\end{array}\right),
\end{aligned}
$$

with $b>0, b \neq 1,-\pi / 3<\theta<\pi / 3$, and $\theta \neq 0$. The four vectors $\left|w_{i}\right\rangle$ read

$$
\begin{aligned}
& \left|w_{0}\right\rangle=|00\rangle+|11\rangle+|22\rangle, \\
& \left|w_{i}\right\rangle=b|i-1, i\rangle+\mathrm{e}^{\dot{\mathrm{i}} \theta}|i, i-1\rangle \quad(i=1,2,3),
\end{aligned}
$$

where the summation is modulo three and $|i\rangle(i=0,1,2)$ form the standard basis in $\mathbb{C}^{3}$. It straightforwardly follows from 10 that this witness has a block-diagonal form $W_{\theta, b}=\Lambda_{\theta}+(1 / b)\left(P_{1}+P_{2}+P_{3}\right)$, where $\Lambda_{\theta}$ is given by

$$
\Lambda_{\theta}=\left(\begin{array}{ccc}
1 & \mathrm{e}^{\dot{\mathrm{i}} \theta} & \mathrm{e}^{-\dot{\mathrm{i}} \theta} \\
\mathrm{e}^{-\dot{\mathrm{i}} \theta} & 1 & \mathrm{e}^{\dot{\mathrm{i}} \theta} \\
\mathrm{e}^{\dot{\mathrm{i}} \theta} & \mathrm{e}^{-\dot{\mathrm{i}} \theta} & 1
\end{array}\right)
$$

and acts on on the subspace spanned by $|i i\rangle(i=0,1,2)$. The three remaining blocks are rank-one matrices $P_{i}=\left|\phi_{i}\right\rangle\left\langle\phi_{i}\right|$ with $\left|\phi_{i}\right\rangle=b|i-1, i\rangle+|i, i-1\rangle(i=1,2,3)$ being vectors spanning a subspace orthogonal to $\Pi_{W_{\theta, b}}$, with the latter, as it is indirectly stated in Ref. [9], consisting of the following six vectors

$$
\begin{aligned}
& \left|z_{1}^{( \pm)}\right\rangle=\left( \pm 1, \omega^{*}, 0\right) \otimes(\omega, \mp 1,0), \\
& \left|z_{2}^{( \pm)}\right\rangle=\left(0, \pm 1, \omega^{*}\right) \otimes(0, \omega, \mp 1), \\
& \left|z_{3}^{( \pm)}\right\rangle=\left(\omega^{*}, 0, \pm 1\right) \otimes(\mp 1,0, \omega),
\end{aligned}
$$


where $\omega=\sqrt{b} \mathrm{e}^{\mathrm{i} \theta / 2}$. Let us finally mention that for any $\theta \in(-\pi / 3, \pi / 3)$ and $\theta \neq 0$, the witness has exactly one negative eigenvalue which comes from $\Lambda_{\theta}$, while for $\theta=0, \Lambda_{0}$ is an unnormalized projector onto $|00\rangle+|11\rangle+|22\rangle$, and therefore $W_{0, b}$ becomes a positive semi-definite matrix for any positive $b$.

We are now ready to show that $W_{\theta, b}$ is not optimal. First of all, one notices that $\Pi_{W_{\theta, b}}$ of $W_{\theta, b}$ consists of only six vectors (14), and therefore $W_{\theta, b}$ does not have the spanning property (cf. Sec. 2). This does not imply, nevertheless, that the witness is not optimal, because examples of optimal decomposable EWs without the spanning property are known [32].

In what follows, exploiting the method sketched in Sec. 2, we will show that $W_{\theta, b}$ is indeed not optimal. To this end, consider a particular pair of vectors from $\Pi_{W_{\theta, b}}$, say $\left|z_{1}^{( \pm)}\right\rangle$. Up to normalization, their local components read $\left|e_{0}^{( \pm)}\right\rangle \propto\left( \pm 1, \omega^{*}, 0\right)$ and $\left|f_{0}^{( \pm)}\right\rangle \propto(\omega, \mp 1,0)$, while vectors orthogonal to both of them can be taken as $\left|e_{1}^{( \pm)}\right\rangle \propto(\mp \omega, 1,0)$ and $\left|e_{2}^{( \pm)}\right\rangle=(0,0,1)$, and $\left|f_{1}^{( \pm)}\right\rangle \propto\left( \pm 1, \omega^{*}, 0\right)$ and $\left|f_{2}^{( \pm)}\right\rangle=(0,0,1)$. It then follows from (7), (8) and (9) that

$$
\begin{aligned}
A_{2}^{( \pm)}\left(W_{\theta, b}\right)=\frac{1}{N_{e} N_{f}} & {\left[2\left(\left|\delta_{1}\right|^{2}+\left|\omega_{1}\right|^{2}\right)+\left(b+\frac{1}{b}-1\right)\left(\left|\delta_{2}\right|^{2}+\left|\omega_{2}\right|^{2}\right)\right.} \\
\pm & \left.\frac{4 \sqrt{b}}{1+b} \sin \left(\frac{3 \theta}{2}\right) \operatorname{Im}\left(\delta_{2} \omega_{2}\right)\right],
\end{aligned}
$$

where $N_{e}=1+\left|\delta_{1}\right|^{2}+\left|\delta_{2}\right|^{2}$ and $N_{f}=1+\left|\omega_{1}\right|^{2}+\left|\omega_{2}\right|^{2}$. It is easily provable that $A_{2}^{( \pm)}\left(W_{\theta, b}\right)$ are strictly positive unless $\delta_{1}=\delta_{2}=\omega_{1}=\omega_{2}=0$. With the aid of the facts that $\operatorname{Im} z \leq|z|$ holds for any $z \in \mathbb{C}$ and $|\sin (3 \theta / 2)| \leq 1$, we can lower bound them in the following way

$$
\begin{aligned}
A_{2}^{( \pm)}\left(W_{\theta, b}\right) \geq \frac{1}{N_{e} N_{f}} & {\left[2\left(\left|\delta_{1}\right|^{2}+\left|\omega_{1}\right|^{2}\right)+\left(b+\frac{1}{b}-1\right)\left(\left|\delta_{2}\right|^{2}+\left|\omega_{2}\right|^{2}\right)\right.} \\
& \left.-\frac{4 \sqrt{b}}{1+b}\left|\delta_{2} \omega_{2}\right|\right],
\end{aligned}
$$

which can later be rewritten as

$$
\begin{aligned}
A_{2}^{( \pm)}\left(W_{\theta, b} \geq_{N_{e} N_{f}}\right. & {\left[2\left(\left|\delta_{1}\right|^{2}+\left|\omega_{1}\right|^{2}\right)+\left(b+\frac{1}{b}-1\right)\left(\left|\delta_{2}\right|-\left|\omega_{2}\right|\right)^{2}\right.} \\
\left.+2\left(b+\frac{1}{b}-1-\frac{2 \sqrt{b}}{1+b}\right)\left|\delta_{2} \omega_{2}\right|\right] &
\end{aligned}
$$

It is not difficult to convince oneself that $b+1 / b-1>1$, and

$$
b+\frac{1}{b}-1-\frac{2 \sqrt{b}}{1+b}>0
$$

for any positive $b \neq 1$; therefore the expression in square brackets in Eq. (17) is always positive except for the case when $\left|\delta_{1}\right|=\left|\delta_{2}\right|=\left|\omega_{1}\right|=\left|\omega_{2}\right|=0$. 

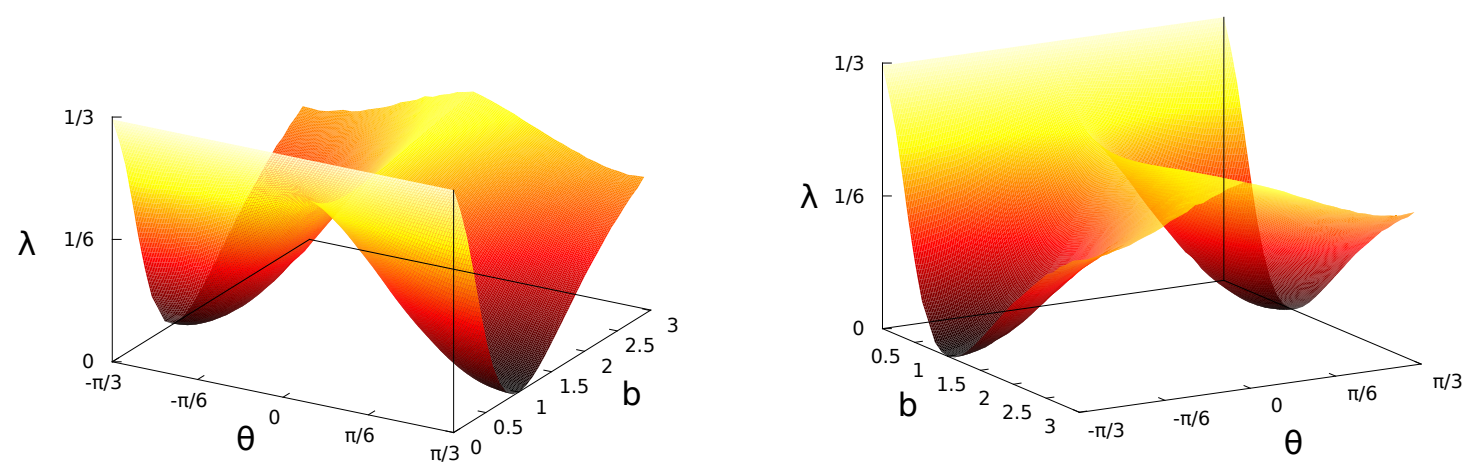

Figure 1. As a function of $\theta \in[-\pi / 3, \pi / 3]$ and $b \in(0,3)$, it is presented the numerically determined maximal value of $\lambda$ such that $\left(\widetilde{W}_{\theta, b}-\lambda \widetilde{P}_{1}\right) /(1-\lambda)$ is an entanglement witness. Here $\widetilde{W}_{\theta, b}$ and $\widetilde{P}_{1}$ denote the normalized witness $W_{\theta, b}$ and the rank-one operator $P_{1}$. The visible maximum for $\theta=0$ is a consequence of the fact that $W_{0, b}$ is a positive matrix for any allowed $b$, and hence one can subtract the whole block $(1 / b) P_{1}$ from it.

For the remaining two pairs of vectors in $\Pi_{W}$, one exploits the fact that $\left|z_{i}^{( \pm)}\right\rangle=$ $S^{i-1} \otimes S^{i-1}\left|z_{1}^{( \pm)}\right\rangle(i=2,3)$ with $S$ being a unitary operator such that $S|i\rangle=|i+1\rangle$ $(i=0,1,2)$, where addition is modulo three. Thus, the vectors orthogonal to the local components of $\left|z_{i}^{( \pm)}\right\rangle$can be taken as $S^{i-1}\left|e_{j}^{( \pm)}\right\rangle$and $S^{i-1}\left|f_{j}^{( \pm)}\right\rangle$with $j=1,2$ (recall that $\left|e_{j}^{( \pm)}\right\rangle$and $\left|f_{j}^{( \pm)}\right\rangle$are vectors orthogonal to the local components of $\left|z_{1}^{( \pm)}\right\rangle$; see above for their explicit forms). One finally checks that

$$
S \otimes S W_{\theta, b} S^{\dagger} \otimes S^{\dagger}=W_{\theta, b} .
$$

All this means that $A_{2}^{( \pm)}\left(W_{\theta, b}\right)$ for both pairs $\left|z_{i}^{( \pm)}\right\rangle(i=2,3)$ is given by the same formula (15). As a consequence, $W_{\theta, b}$ is not optimal for any positive $b \neq 1$ and $|\theta| \in(0, \pi / 3)$, and hence cannot serve as a counterexample to the conjecture.

With the aid of the procedure described in Ref. [33] we have also numerically studied optimality of the entanglement witness (10). Since the optimization over product vectors may lead to different local minima, several initial conditions, uniformly and randomly chosen have been considered, and this procedure was repeated at least $10^{3}$ times for each choice of $\theta$ and $b$ (the increment step was taken as 0.05 and 0.1 , respectively). It clearly follows from Fig. 1 that the obtained results fully support the above proof. As a positive operator to be subtracted from the witness we took $\widetilde{P}_{1}=P_{1} /\left(1+b^{2}\right)$ (see above for the definition of $P_{1}$ ). Then, as a function of $\theta \in[-\pi / 3, \pi / 3]$ and $b \in(0,3)$ we determined the maximal value of $\lambda$ such that $\widetilde{W}(\lambda)=\left(\widetilde{W}_{\theta, b}-\lambda \widetilde{P}_{1}\right) /(1-\lambda)$ is an entanglement witness (see Fig. 1), where $\widetilde{W}_{\theta, b}$ is a normalized version of $W_{\theta, b}$. It should be noticed that $W_{0, b}$ is a positive matrix and therefore one can remove the whole block $(1 / b) P_{1}$ from it. This corresponds to the fact that for any fixed $b$, the function on Fig. 1 achieves its maximum for $\theta=0$. 


\section{Concluding remarks}

We have shown in this note, exploiting the method formulated in Ref. [13, that the decomposable entanglement witness introduced in Refs. [9, 10] is not optimal. This in particular means that, contrary to the claim of [9], it does not disprove the SPA conjecture in the decomposable case. Nevertheless, a proper counterexample has recently been found by other researchers [12]. This together with an analogous counterexample in the indecomposable case provided by Ha and Kye [7], fully disproves the SPA conjecture.

Still the fact that SPAs of some optimal positive maps are entanglement breaking is interesting because one of its main advantages is that the physical implementation of the resulting completely positive maps can be simplified to a measure \& prepare scheme (see [1]). Therefore, it is reasonable to ask as to whether the notion of SPA can be modified so that the resulting completely map "best approximating" a given positive map is entanglement breaking [5, 6]. And, actually, it was already shown in Ref. [5] that for any optimal decomposable map there exists an entanglement breaking channel (not necessarily the fully depolarizing one) such that their mixture with the smallest possible mixing parameter necessary to obtain a completely positive map, is another entanglement breaking channel. In the language of entanglement witnesses this means that for any optimal decomposable witness there is a separable state such that their combination with the smallest "weight" necessary to turn the obtained operator into a positive one, is a separable state. And, the obtained approximation is the best in the sense that the resulting operator lies on the boundary of the convex set of positive operators. In the indecomposable case, however, the question is left open.

\section{Acknowledgments}

Discussions with J. Stasińska are acknowledged. This work is supported by the ERC Grant QUAGATUA, EU projects AQUTE and SIQS, and Spanish TOQATA. R. A. acknowledges support from the Spanish MINECO through the Juan de la Cierva program. J. B. acknowledges National Research Foundation and Ministry of Education of Singapore.

\section{References}

[1] Korbicz J K, Almeida M L, Bae J, Lewenstein M and Acín A 2008 Phys. Rev. A 78062105.

[2] Chruściński D, Pytel J and Sarbicki G 2009 Phys. Rev. A 80062314.

[3] Chruściński D and Pytel J 2010 Phys. Rev. A 82052310.

[4] Chruściński D and Pytel J 2011 J. Phys. A: Math. Theor. 44165304.

[5] Augusiak R, Bae J, Czekaj Ł and Lewenstein M 2011 J. Phys. A: Math. Theor. 44, 185308.

[6] Hansen L O, Hauge A, Myrheim J and Sollid P Ø 2013 Extremal entanglement witnesses, arXiv: 1305.2385 .

[7] Ha K-C and Kye S-H 2012 J. Math. Phys. 53102204.

[8] Størmer E 2013 J. Funct. Anal. 2642197. 
[9] Ha K-C and Kye S-H 2012 Separable states with unique decompositions, arXiv:1210.1088v3.

[10] Ha K-C and Kye S-H 2012 Separable states with unique decompositions, to be published in Comm. Math. Phys. (arXiv:1210.1088v5).

[11] Augusiak R, Bae J, Tura J and Lewenstein M Comment on Separable states with unique decompositions, arXiv:1304.2040 1 .

[12] Chruściński D and Sarbicki G 2013 Disproving the conjecture on structural physical approximation to optimal decomposable entanglement witnesses, arXiv:1309.2883.

[13] Lewenstein M, Kraus B, Cirac J I and Horodecki P 2000 Phys. Rev. A 62052310.

[14] Werner R F 1989 Phys. Rev. A 404277.

[15] Horodecki R Horodecki P Horodecki M and Horodecki K 2009 Rev. Mod. Phys. 81865.

[16] Gühne O and Tóth G 2009 Phys. Rep. 4741.

[17] Horodecki M, Horodecki P and Horodecki R 1996 Phys. Lett. A 2231.

[18] Terhal B M 2000 Phys. Lett. A 271319.

[19] Augusiak R, Tura J and Lewenstein M J. Phys. A: Math. Theor. 44212001.

[20] Skowronek Ł, Størmer E and Życzkowski K 2009 J. Math. Phys. 50062106.

[21] Ha K-C and Kye S-H 2011 Open Syst. Inf. Dyn. 18323.

[22] Chruściński D and Sarbicki G 2012 J. Phys. A: Math. Theor. 45115304.

[23] Jamiołkowski A 1972 Rep. Math. Phys. 3275.

[24] Choi M-D 1980 Positive Linear Map, Operator Algebras and Applications, Kingston, 1980, Proc. Sympos. Pure Math., vol. 38. Part 2, Amer. Math. Soc., 1982, pp. 583-590.

[25] Paulsen V Completely Bounded Maps and Operator Algebras, Cambridge Studies in Advanced Mathematics vol. 78, Cambridge: Cambridge University Press, 2002.

[26] Holevo A. S 1999 Russian Math. Surveys 531295 (arXiv:quant-ph/9809023).

[27] Horodecki M, Shor P W and Ruskai M B 2003 Rev. Math. Phys. 15629.

[28] Størmer E 1963 Acta Math. 110233.

[29] Woronowicz S L 1976 Rep. Math. Phys. 10165.

[30] Walgate J and Scott A J 2008 J. Phys. A: Math. Theor. 41375305.

[31] Horodecki P 2003 Phys. Rev. A 68052101.

[32] Augusiak R, Sarbicki G and Lewenstein M 2011 Phys. Rev. A 84052323.

[33] Leinaas J M, Myrheim J and Sollid P Ø2010 Phys. Rev. A 81062329. 